\title{
Prediction the Performance of the IAEA 10 MW MTR Research Reactor at Transient Startup
}

\author{
Magy. M. Kandil \\ Nuclear and Radiological Regulatory Authority, Cairo, Egypt
}

\begin{tabular}{l} 
ART ICLE INFO \\
\hline Article history: \\
Received: $20^{\text {th }}$ Jul. 2020 \\
Accepted: $18^{\text {th }}$ Mar. 2021 \\
\hline
\end{tabular}

Keywords:

IAEA 10 MW MTR Research

Reactor, Startup,

Thermal-Hydraulic,

Neutron Flux,

Fuel Temperature,

Coolant Temperature.

\begin{abstract}
In this research, a new dynamic model is developed for the IAEA 10 MW MTR as a research reactor. The developed dynamic model predicts the performance of the core design and the thermal hydraulic behavior in reactivity variations conditions for controlling startup conditions to reach criticality. The startup is performed by increasing or decreasing the reactivity due to insertion or withdrawal of a control rod with/without an external neutron source. The developed model is a dynamic model made by MATLAB/SIMULINK with a systematic solution of a combination of Ordinary Differential Equations (ODE) of Point Kinetic Equation (PKE) and the thermal hydraulic equations to calculate the change of neutron density, fuel and cooling temperatures through reaching the critical state before a new reactivity is added. The developed dynamic model predictions are compared with the calculations performed in various establishments using other codes. The results showed that the developed model can provide accurate predictions during transient conditions; and can be used for analyzing the lowest power reactors encountered in practice.
\end{abstract}

\section{1- INTRODUCTION}

In zero-power reactors, the neutron flux density must be controlled, since it is proportional to the reactor power [1]. Ordinarily, these reactors do not virtually contain (or contains very small) number of free neutrons during shut down. Starting up a nuclear reactor needs resources to generate a controlled nuclear fission chain reaction clearly and safely and continuously. The total reactivity in the reactor system dependent on lifting the control rods discontinuously. At startup, the control rods are withdrawn at time intervals thus the reactivity is presented linearly in the reactor core, to allow criticality to be reached in a slow and safe manner [2]. In nuclear reactors, there is a time delay through displaying the neutron flux density changes that may cause the operator to evaluate the reactor state wrongly and probably to increase the multiplication factor to disallowed supercritical series [2]. The following fundamentals guarantee a safe reactor startup: the reactor must be started from an effectively value of the neutron flux density; the initial neutron flux density must be raised up such that the neutron detectors carry appropriate values, thus, external neutron sources are used for controlling the subcritical condition in nuclear reactors [3]. The following fundamentals guarantee a safe reactor startup: the reactor must be started from an effectively value of the neutron flux density; the initial neutron flux density must be raised up such that the neutron detectors carry appropriate values, thus, external neutron sources are used for controlling the subcritical condition in nuclear reactors [3]. Ziya Akcasu et al, [4] and other researches have solved the point kinetics equations analytically for any ramp reactivity insertion to reach the criticality of the reactor without considering the external neutron source in power contribution., and others such as Zhang et al [5] also used the one group model in arising the analytical solution of point kinetics equation with external neutron source, but the solution is useful when the reactivity is linearly introduced discontinuously and not in other types of effects such as transient ramp reactivity due to rod insert or withdraw at startup conditions.

In nuclear reactors, the startup external sources of neutrons are other than delayed fission or prompt 
neutrons since they come from sources other than neutron-induced fission[5]. The startup neutron sources are typically injected in frequently spaced positions in the reactor core, in many of the fuel rods. These sources are important for safe reactor startup because the impulsive fission and cosmic rays assist as weak neutron sources, that are too weak for the reactor instrumentation deductions; depend on them could a chief to a "blind" start, which is a possibly become unsafe condition. The neutron sources are herefore positioned. Consequently, the neutron flux they produce is always detectable by the reactor instrumentations.

To date, it has been difficult to settle down standardized computer tools and procedures for the safety analysis of nuclear power plants since they consist of many system-of-systems as established through their internal interdependent systems. The negative impact of such interdependence importance was demonstrated after the 2011 Fukushima Daiichi nuclear disaster. Hence, there is a major need for new strategies to overcome the limitations of the existing risk assessment techniques such as the use of fault tree and static event schemes, through principally completed by simulation of the nonlinear dynamic feedback mechanisms among the different NPP systems/components. This could be due to the big differences in nuclear reactors power densities, large variety of design and fuel geometries, different modes of operations, purposes of utilization, etc. In general, there was a great effort for development of proper tools and codes for each nuclear reactor. Furthermore, these dedicated codes are generally based on conservative physical models and are intended to focus on specific phenomena in the reactor.[6]

Thus, in this research, a new dynamic model is developed for simulating the Point Kinetic Equations and thermal-hydraulics for any research reactor to predict the whole changes of the neutron flux, the fuel temperature and the cooling temperature at startup with ramp reactivity increasing due to an abnormal withdrawal of a control rod condition with/without an external of neutron source. The new developed model is applied in Material Testing Reactors (MTR) (10MW IAEA) as a research reactor. For validation of the developed modeling, comparison between this work and other analytical solutions of point reactor kinetic equations for one-group of delayed neutrons is accomplished with computed the change in neutron density as soon as reactivity is increased introduced unevenly. The comparison is applied with the solutions using at eidi et al. [7], Reisch et al. [8] and Zhang et al.[5].

\section{2- The Developed Dynamic Model}

The dynamic model is developed using MATLAB/SIMULINK as shown in Figure (1). This model is based on; reactor point kinetics, with six groups of delayed neutrons, thermal hydraulic, one dimensional heat conduction created with lumped parameters and the feedback due to the; fuel and coolant temperature effects. Core calculations have been performed by a new developed supporting software algorithm which is able to calculate neutronic and thermal hydraulic parameters of any low power reactor core. The developed model used to predict behavior of the core using Ordinary Differential Equations (ODEs) under steady state and transient startup conditions [9] with/without External source are applied on the MTR 10 MW IAEA typical reactor core.

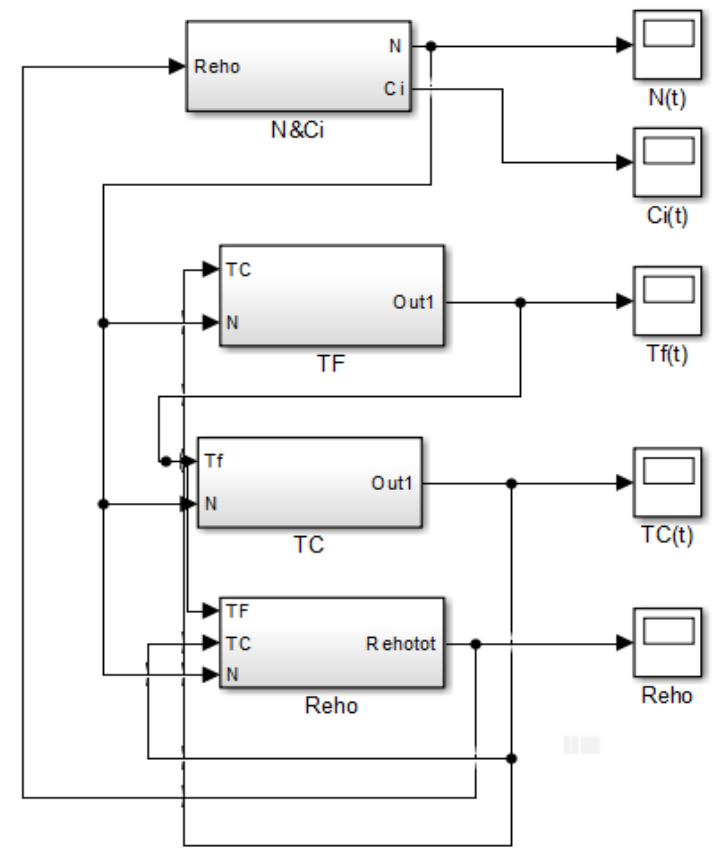

Fig. (1): The main Simulink of the developed dynamic model

\subsection{The Core neutronic calculations in the developed model}

The point kinetics equation used in the core module is referred to byReisch [8]. The equation system is solved numerically with the new developed dynamic modeling and applied during transient operations, when the fuel and coolant temperature increase, are considered in reactivity calculations. The point 
kinetics equations are stated in relationships of reactor neutron flux $(N)$ which is proportional to the reactor power $(P)$ and the average neutron density with the stability equations as:

$$
\begin{aligned}
& \frac{d N}{d t}=\frac{\rho(t)-\beta}{\Lambda} N(t)+\sum_{i}^{6} \lambda_{i} C_{i}(t)+q(t) \\
& \frac{d C_{i}}{d t}=\frac{\beta_{i}}{\Lambda} N(t)-\lambda_{i} C_{i}(t)
\end{aligned}
$$

Where: $\mathrm{t}$ is time (sec), $N$ is neutron flux, $\rho(t)$ is the neutron Reactivity, $\beta$ is sum of the delayed neutron fractions, $\beta_{i}$ is the $\mathrm{i}^{\text {th }}$ delayed neutron fraction, $\Lambda$ is neutron mean lifetime, $\lambda_{i}$ is $i^{\text {th }}$ decay constant for the delayed neutron precursor. $C_{i}$ is concentration of the $\mathrm{i}^{\text {th }}$ fraction of the delayed neutrons, precursors, and $q$ is the external neutron source.

During steady-state operation, the reactivity in equation (1) is time dependent even still it is zero, all time derivatives are equal to zero, the initial value of the relative neutron flux equals unity $\left(\mathrm{N}_{0}=1\right)$. From equation (2) at $\mathrm{t}=0, C_{i}$ can be determined as following:

$$
\begin{aligned}
& \frac{\mathrm{dC}_{\mathrm{i}}}{\mathrm{dt}}=0 \\
& \mathrm{c}_{\mathrm{i} 0}=\frac{\beta_{\mathrm{i}}}{\lambda_{\mathrm{i}} * \Lambda} \mathrm{N}_{0}
\end{aligned}
$$

\subsection{External neutron source contribution}

\subsubsection{Continuous external neutron source}

The neutron density is calculated with intended constant source as in equation (5),

$$
\mathrm{q}_{\mathbf{n}}=\mathrm{q}_{\mathbf{0}}
$$

\subsubsection{Time variation external neutron source}

The neutron density is determined for distinction of the external neutron source with respect to time is considered as flowing:

\subsubsection{The external neutron source as polynomial of time}

The external neutron source is well-defined by means of the ratios of polynomials of degree $j[8]$ :

$$
\mathrm{q}(\mathrm{t})=\sum_{\mathrm{n}=0}^{\mathrm{j}} \mathrm{q}_{\mathrm{n}} \mathrm{t}^{\mathrm{n}}
$$

$$
\rho(t)=\left\{\begin{array}{cr}
\rho_{0}+\text { rt } & 0 \leq t<\text { to } \\
\rho_{0}+\text { rto } & t \geq \text { to }
\end{array}\right\}
$$

Where: $\rho_{0}$ is the initial subcritical reactivity, $\mathrm{r}$ represents the ramp reactivity count rates and to is the time for withdrawn the rods

\subsubsection{The external neutron source as Sinusoidal fluctuation with time variation}

Due to fluctuation of neutron source around a constant value, the source is time dependent. Accounting for the sinusoidal external neutron source that is welldefined by accounting for the sinusoidal function as shown in equation (8).

$$
\mathrm{q}_{\mathrm{n}}=\mathrm{q}_{0} \sin \omega \mathrm{t}\left(\omega=\frac{2 \pi}{\mathrm{T}}\right)
$$

where $\omega$ and $\mathrm{T}$ are angular frequency and period of source, similarly that can be suitable for indicating the situation effects in the neutron density [9].

\subsection{The Reactivity calculations}

\subsubsection{The Reactivity as of Fuel's Effect}

The Reactivity from Fuel's Effect dependents on the fuel temperature changing $\left(T_{F}\right)$ after the power changes with a time delay $\left(\tau_{F}\right)$ :

$$
\mathrm{T}_{\mathrm{F}}=\frac{\mathrm{C}_{\mathrm{FN}} \mathrm{N}}{1+\frac{\mathrm{d}}{\mathrm{dt}} \tau_{\mathrm{F}}}
$$

Where: $T_{F}$ is the Fuel temperature change, $\mathrm{C}_{\mathrm{FN}}$ is the fuel temperature proportionality constant to the neutron flux, $\tau_{F}$ isthe thermal time constant of the fuel, $\mathrm{t}$ is the time.

From equation (9), the differential equation for the Fuel temperature predicted over time is:

$$
\begin{aligned}
& \mathrm{T}_{\mathrm{F}}+\tau_{\mathrm{Fuel}} \frac{\mathrm{dT}_{\mathrm{F}}}{\mathrm{dt}}=\mathrm{C}_{\mathrm{FN}} \mathrm{N} \\
& \frac{\mathrm{dT}_{\mathrm{F}}}{\mathrm{dt}}=\frac{\mathrm{C}_{\mathrm{FN}}}{\tau_{\mathrm{F}}} \mathrm{N}-\frac{1}{\tau_{\mathrm{F}}} \mathrm{T}_{\mathrm{F}}
\end{aligned}
$$

The reactivity of the Fuel's Effect is:

$$
\delta \rho_{\mathrm{F}}=\alpha_{\mathrm{F}}\left(\mathrm{T}_{\mathrm{F}}-\mathrm{T}_{\mathrm{F} 0}\right)
$$

Where: $\delta \rho_{F}$ is the reactivity due to the fuel temperature change and $\alpha_{F}$ is the fuel temperature coefficient. At the initial time $(\mathrm{t}=0)$ and at steady state $\delta \rho_{F 0}=0$

Their reactivity is denoted by: 


\subsubsection{The Reactivity as of Coolant's Effect}

The Reactivity results from the coolant's effect dependents on the changing of the Coolant Temperature. As fuel equations (10\&11), the coolant differential equations are like to that of the fuel but the coolant thermal time constant is higher [8]:

$$
\begin{aligned}
& \mathrm{T}_{\mathrm{C}}+\tau_{\mathrm{C}} \frac{\mathrm{dT}_{\mathrm{C}}}{\mathrm{dt}}=\mathrm{C}_{\mathrm{CN}} \\
& \frac{\mathrm{dT}_{\mathrm{C}}}{\mathrm{dt}}=\frac{\mathrm{C}_{\mathrm{CN}}}{\tau_{\mathrm{C}}} \mathrm{N}-\frac{1}{\tau_{\mathrm{C}}} \mathrm{T}_{\mathrm{C}}
\end{aligned}
$$

Where: $T_{C}$ is the coolant temperature change, $\tau_{C}$ is the Coolant thermal time constant, $C_{C N}$ is the coolant temperature proportionality constant to the relative neutron flux.

Assuming that at zero power operation coolant reactivity contribution from temperature change:

$$
\delta \rho_{\mathrm{C}}=\alpha_{\mathrm{C}}\left(\mathrm{T}_{\mathrm{C}}-\mathrm{T}_{\mathrm{C} 0}\right)
$$

where: $\delta \rho_{C}$ is the reactivity due to the coolant temperature change and $\alpha_{C}$ is the coolant temperature coefficient. At the initial time $(\mathrm{t}=0)$ and at steady state $\delta \rho_{C 0}$ is zero,

\subsubsection{The Reactivity of the Control Rods}

Differential control rod worth is defined as the reactivity change per unit movement of the control rod and it is generally expressed in $\mathrm{pcm} / \mathrm{cm}$ that defined as:

$\delta \rho_{R}$ is the reactivity of the control rods' movement

\subsubsection{The Total Internal Reactivity of the model}

The internal reactivity balance with the control rods, the Fuel's and the Coolant's temperature effect is:

$$
\delta \rho=\delta \rho_{\mathrm{R}}+\delta \rho_{\mathrm{F}}+\delta \rho_{\mathrm{C}}
$$

Where: $\delta \rho_{\mathrm{F}}$ is the reactivity of the fuel

$\delta \rho_{\mathrm{C}}$ is the reactivity of the coolant

$\delta \rho_{\mathrm{R}}$ is the reactivity of changing the control rods' movement

\section{3 - The methodology}

The dynamic model is developed based on; reactor point kinetics, through using equations (1) \& (2) and from (11), (14) and (16) as a system of coupled linear ordinary and thermal-hydraulic differential equations for representing the Time-dependent parameters of reactor system (the reactivity and the neutron source terms.

\subsection{Appling the Developed Model on 10MW IAEA MTR typical reactor core}

The developed dynamic modeling is applied for IAEA $10 \mathrm{MW}$, the above equations define completely the model with, following specification of initial values that represented in table $1 \& 2$ [8].

Table (1): The Decay constants, yields of delayedneutron precursors in the thermal fission of uranium 235 and the initial values of the delayed neutrons' precursors

\begin{tabular}{cccc}
\hline $\mathrm{G}$ & $\boldsymbol{\lambda}_{\boldsymbol{i}},\left[\mathrm{s}^{-1}\right]$ & $\boldsymbol{\beta}_{\boldsymbol{i}}$ & $\mathbf{C}_{\boldsymbol{i}}=\frac{\boldsymbol{\beta}_{\boldsymbol{i}}}{\boldsymbol{\lambda}_{\boldsymbol{i}} * \boldsymbol{\Lambda}} * \mathbf{N}_{\mathbf{0}}$ \\
\hline 1 & 0.0124 & 0.000215 & 17.3387 \\
2 & 0.0305 & 0.00142 & 46.6885 \\
3 & 0.1110 & 0.00127 & 11.4775 \\
4 & 0.3010 & 0.00257 & 8.5316 \\
5 & 1.1300 & 0.00075 & 0.6561 \\
6 & 3.0000 & 0.00027 & 0.0907 \\
\hline
\end{tabular}

Table (2): The initial values and parameters of the developed dynamic model parameters

\begin{tabular}{cc}
\hline Parameters & values \\
\hline $\mathbf{T}_{\mathbf{F} \mathbf{0}}$ & 0.001 \\
$\mathbf{T}_{\mathbf{C 0}}$ & 0.0005 \\
$\mathbf{N}_{\mathbf{0}}$ & 1 \\
$\mathbf{q}_{\mathbf{0}}$ & $10^{9}$ \\
$\boldsymbol{\Lambda}$ & $0.001 \mathrm{sec}$ \\
$\boldsymbol{\tau}_{\mathbf{F}}$ & $5 \mathrm{sec}$ \\
$\boldsymbol{\tau}_{\mathbf{C}}$ & $100 \mathrm{sec}$ \\
$\mathbf{C}_{\mathbf{F N}}$ & 0.001 \\
$\mathbf{C}_{\mathbf{C N}}$ & 0.0005 \\
$\boldsymbol{\alpha}_{\mathbf{F}}$ & $-3.1 \times 10^{-5}$ \\
$\boldsymbol{\alpha}_{\mathbf{C}}$ & $-0.6 \times 10^{-5}$ \\
\hline
\end{tabular}




\section{4- THE RESULTS \& DISCUSSION}

The results of the developed model for $\quad r_{-} 0=$ $0.0001 \mathrm{~s} \_1, \rho \_0=-0.006, \mathrm{q} \_0=109$ neutron. $\mathrm{Cm}-3 \mathrm{~s}-1$, and $\mathrm{t} \_0=10 \mathrm{~s}$ and $0 \leq \mathrm{t} \leq 100 \mathrm{~s}$ with different forms of external neutron source with/without considered feedback reactivity of fuel and cooling. The neutron densities results for the insertion of ramp reactivity due to move the control rod from $50 \mathrm{pcm}$ to $60 \mathrm{pcm}$ in the existence of a constant external neutron source, a polynomial and a Sinusoidal in the following sections:

\subsection{Constant External Neutron Source}

Figures $2(a, b)$ shows the results of the developed model in the existence of a constant external neutron source with/without considered feedback reactivity of fuel and cooling temperatures.

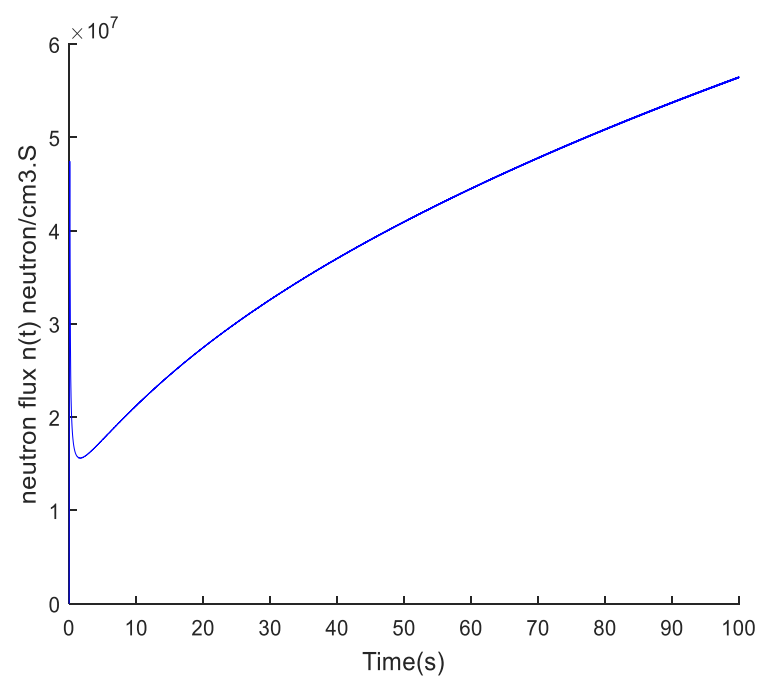

(a)

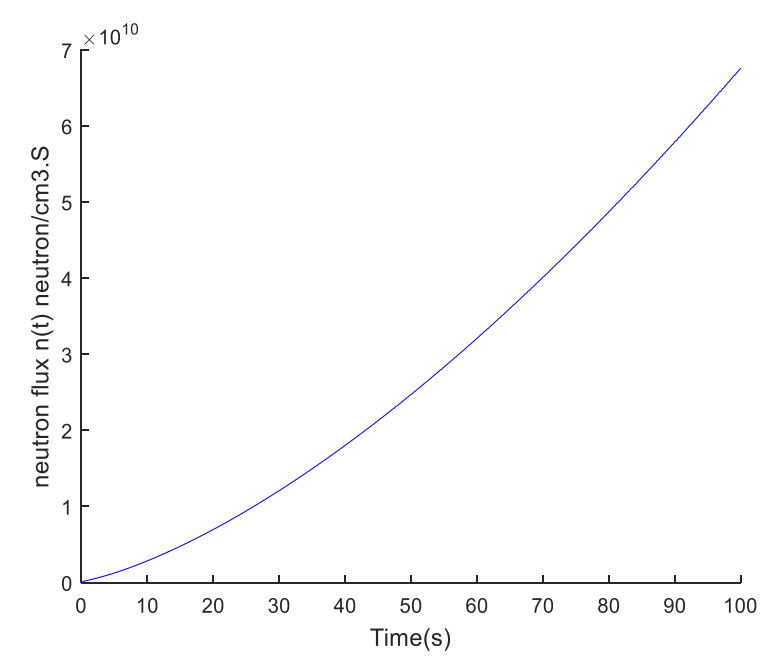

(b)

Fig. (2: a, b): The neutron flux with Constant External Neutron Source

\subsection{Variable external neutron source with time}

Figures $3(a, b)$ and $4(a, b)$ show the results of the developed model in the existence of external neutron source of a polynomial and Sinusoidal external neutron source of time with/without considered feedback reactivity of fuel and cooling at transient startup respectively.

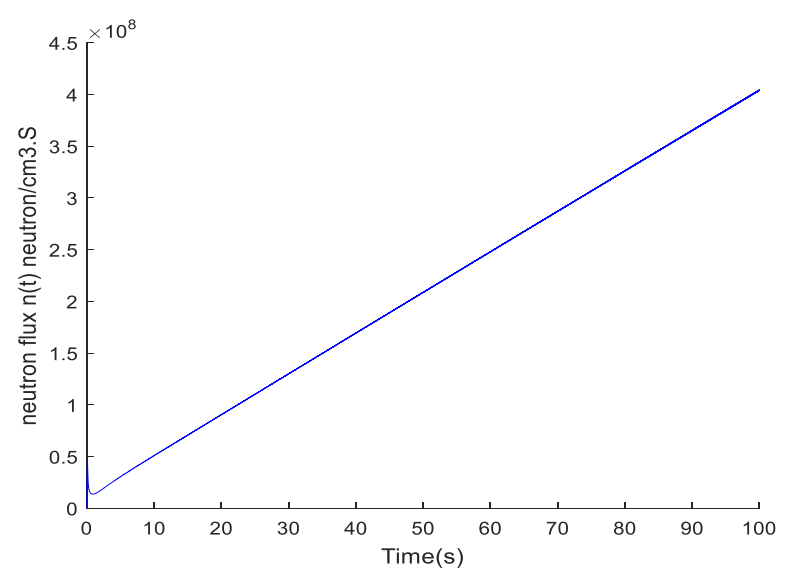

(a)

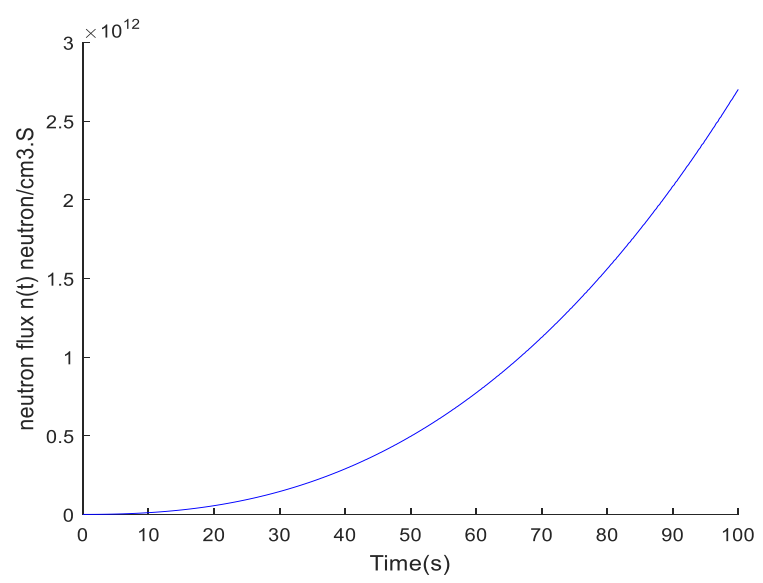

(b)

Fig. (3: a, b): The neutron flux with external neutron source as polynomial of time

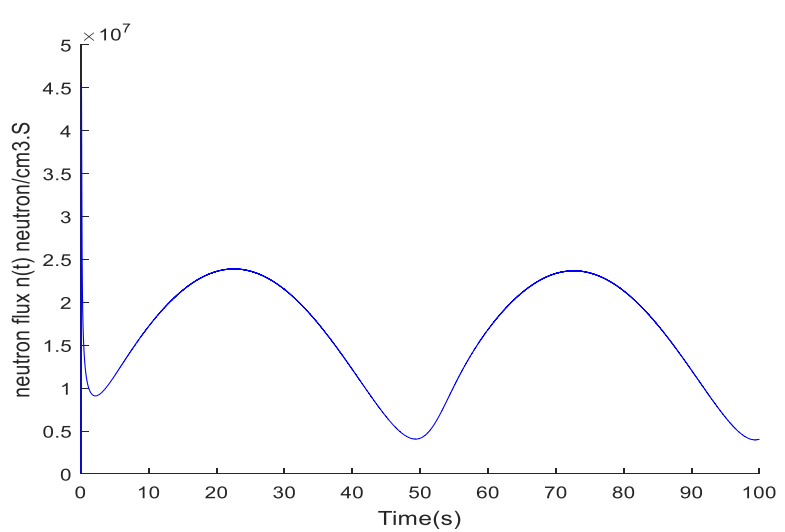

(a) 


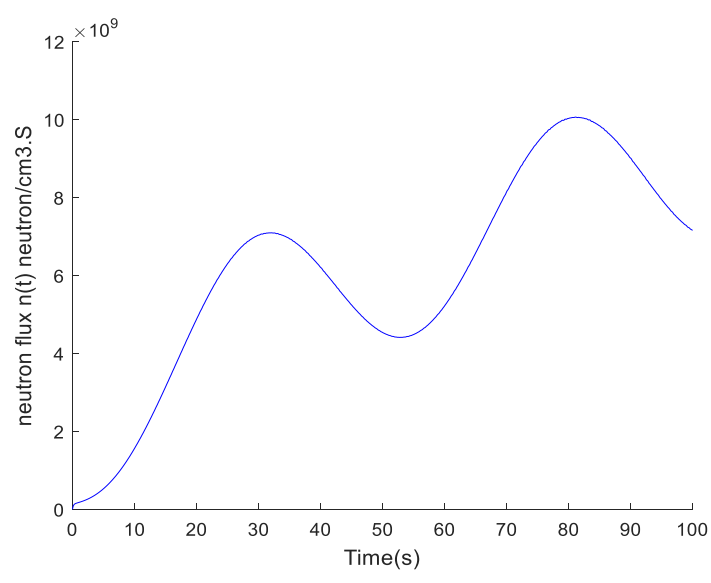

(b)

Fig. (4: a,b): The neutron flux with a Sinusoidal external neutron source

From previous figures 2,3 and 4 the variation of neutron density during startup in the presence of all types of the external neutron source, increased with large values without feedback more than with feedback. Thus, the nuclear reactor must be considered the feedback reactivity of fuel and cooling temperatures during modeled. The sinusoidal neutron source gave more fluctuation so it is preferred.

\section{5- Validation the developed dynamic Model}

\subsection{Validations without External neutron source}

The validity of the developed dynamic model was tested by comparing the obtained results with the other published model [8]. The comparison has shown in figure 5 demonstrated that the output of the relative neutron flux, the adequacy of the developed dynamic model for investigating transient startup conditions when control rod move from 60 to $70 \mathrm{pcm}$ without external neutron source for predicting the core behavior for IAEA $10 \mathrm{MW}$ as research reactor.

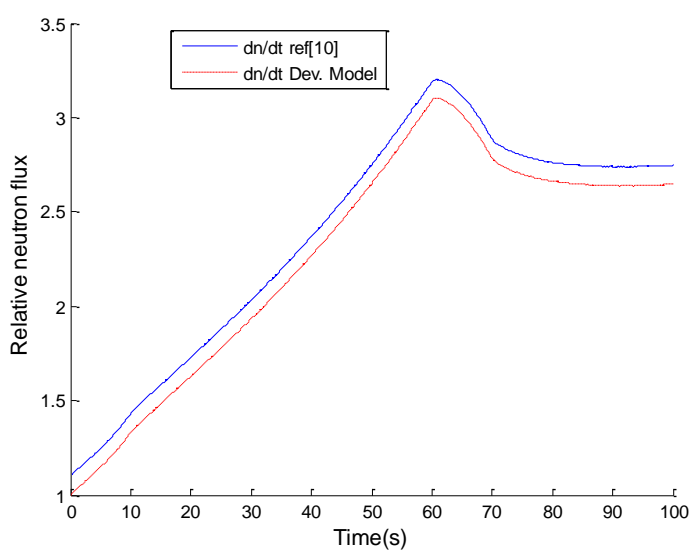

Fig. (5): The change neutron flux without external source at Transient from 60 to $70 \mathrm{pcm}$
The results showed that the developed model can provide accurate predictions during transient conditions; and can be used for analyzing the lowest power reactors encountered in practice as references [8].

\subsection{Validation with External neutron source}

Yamoah, et al. [9] established an analytical expression for the calculation of neutron density response created with the kinetics equations with one-group of delayed neutron precursors as eq $1 \& 2$, by assuming the prompt jump approximation and a constant neutron source, [9] produced the following expression for the neutron density:

$$
n(t)=\left\{\begin{array}{cc}
\frac{\beta n_{0}+q A}{\beta+\left|\rho_{0}\right|-r t^{\prime}} & 0 \leq t<t_{0} \\
\frac{q A}{\left|\rho_{0}\right|-r t_{0}}\left[1-e^{-\frac{\lambda\left[\left|\rho_{0}\right|-r t_{0}\right]}{\beta+\left|\rho_{0}\right|-r t_{0}}}\left(t-t_{0}\right)\right. & \\
\frac{\beta n_{0}+q A}{\beta+\left|\rho_{0}\right|-r t_{0}} e^{-\frac{\lambda\left[\left|\rho_{0}\right|-r t_{0}\right]}{\beta+\left|\rho_{0}\right|-r t_{0}}}\left(t-t_{0}\right) & , t \geq t_{0}
\end{array}\right.
$$

where, $q$ is the external neutron source intensity, $r$ is the velocity of inserted reactivity, $t$ is time, $t 0$ is the duration of each lifting of control rod and $\rho 0$ is the subcritical reactivity.

In order to verify the developed model proposed in this study for the prediction of the neutron density distribution, compare with [9] as numerical solution of the point kinetics equations, Eq. (17). Figure 5 shows the results of Comparison between the developed model and the method perform at [9] for neutron density response $r_{0}=0.0001 \mathrm{~s}^{-1}, \rho_{0}=-0.006, \mathrm{q}_{0}=10^{9}$ neutron. $\mathrm{Cm}^{-3} \mathrm{~s}^{-1}$ and $t_{0}=5 \mathrm{~s}$ and $0 \leq t \leq 80 \mathrm{~s}$.

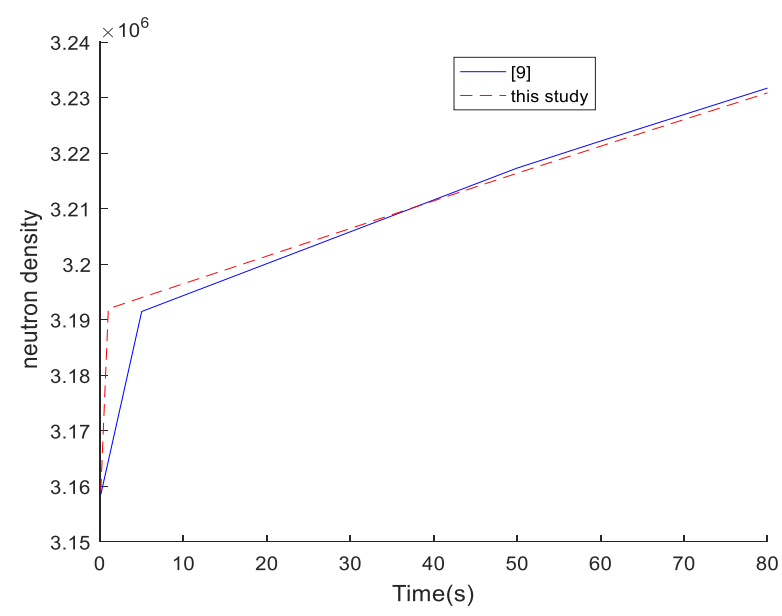

Fig. (6): Comparison between the neutron density response the developed model and the method perform at [9] 


\section{6- CONCLUSION \& FUTURE WORK}

The external of neutron source is used to increase the availability of monitoring the reactivities to control the core parameters during withdrawing or inserting control rods in the core at the subcritical conditions. In this research, a new dynamic modeling is developed for predicted the startup behavior of zero power nuclear reactor in exitance/no exitance external neutron sources with different forms. The new model is developed using a lumped parameters approach for the coupled kinetics and thermal-hydraulics, with/without continuous reactivity feedback due to coolant and fuel temperature effects. The developed dynamic model is applied on IAEA $10 \mathrm{MW}$ MTR as Research Reactor (zero power). The developed dynamic model is useful, where it permits to predict with simply means the dynamic response of research reactor cores. The developed model incorporates the random nature of the source and linear reactivity variation and it is using a general relationship between the expectation values of source intensity and the expectation values of neutron density of the subcritical reactor. The results of the developed model represented that it can be predicted as zero power reactors such as in references [8]. Moreover, it has a good agreement with the results obtained with numerical solution in [9]. The developed model can be changed to use in future for another nuclear reactors such as Pressurized Water Reactors and Boiling Water Reactors.

\section{REFERENCES}

[1] G. Stein, C. Dubi , "Stabilization of the stochastic point reactor kinetic equation through power feedback", Eur. Phys. J. Plus (2020) 135:208, 3 February 2020. https://doi.org/10.1140/epjp/s13360-020-00215-z.

[2] S. M. Ibrahim a , S. E. El-Morshedyb , A. Abdelmaksoud, "Thermal hydraulic analysis of core flow bypass in a typical research reactor", ScienceDirect Nuclear Engineering and Technology journal, Oct. (2018). homepage: www.elsevier.com/locate/net

[3] U. F. Alves1, L. S. Castrillo F. A. Lima, "Insertion Of Reactivity (RIA) Without Scram in The Reactor
Core Iea-R1 Using Code Paret", International Nuclear Atlantic Conference - INAC ,2013.

[4] ZiyaAkcasu, Gerald S. Lellouche, and Louis M. Shotkin, "Mathematical Methods in Nuclear Reactor Dynamics", (New York: Academic Press, 1971, 460 pp.)

[5] Zhang, F., W.Z. Chen and X.W. Gui, "Analytic method study of point-reactor kinetic equation when cold start-up". Ann. Nucl. Energ., 35: 746 749. 2008. world journal of nuclear science and technology.

[6] Martina Adorni, Anis Bousbia-Salah, Francesco D'auria, Tewfik Hamidouche, "Analysis Of Rod Ejection Accident In A Research Reactor By The Coupled Code Technique", Conference: RRFM2011, March 2011, Rome, Italy.

[7] M. Seidi, S. Behnia, R. Khodabakhsh," Generalization of the analytical solution of neutron point kinetics equations with time-dependent external source", open access at Springer link.com, Sep, 2014.

[8] F. Reisch, "Reactor Kinetics Equations applied to the start-up phase of a Ringhals PWR", Ens news, 2006.

[9] S. Yamoah, E. Akaho, B. Nyarko, M. Asamoah and P. Asiedu-Boateng, "Analytical Solution of the Point Reactor Kinetics Equations for One-Group of Delayed Neutrons for a Discontinuous Linear Reactivity Insertion", Research Journal of Applied Sciences, Engineering and Technology 4(21): 4320-4324,2012.

https://www.researchgate.net/publication/26593980 6_Analytical_Solution_of_the_Point_Reactor_Kine tics_Equations_for_One-

Group_of_Delayed_Neutrons_for_a_Discontinuous _Linear_Reactivity_Insertion

[10] Technical University Dresden, Institute of Power Engineering Training Reactor, "Reactor Training Course Experiment “, "Reactor Start-up Procedure". https://turesden.de/ing/maschinenwesen/iet/wket/ ressourcen/dateien/akr2/Lehrmaterialien/start_e.p df?lang=en 